\title{
PEUTZ JEGHERS SYNDROME - A CASE REPORT
}

Rajshekar S. J., S. S. Inamdar, S. K. Andola, Ashraf A. Z.

1. Professor, Department of Pathology, M. R. Medical College, Gulbarga.

2. Professor, Department of Pathology, M. R. Medical College, Gulbarga.

3. Professor \& HOD, Department of Pathology, M. R. Medical College, Gulbarga.

4. Post Graduate, Department of Pathology, M. R. Medical College, Gulbarga.

\section{CORRESPONDING AUTHOR}

\section{Dr. Rajshekar S. J.,}

Department of Pathology,

M. R. Medical College, Gulbarga,

Karnataka

E-mail: sai.drrsj@gmail.com

Ph: 00919035247898.

ABSTRACT: Peutz-Jeghers syndrome is an autosomal dominantly inherited syndrome characterized by mucocutaneous pigmentation and gastrointestinal polyposis. In addition to an elevated risk of gastrointestinal cancers, an increased risk of cancers at other sites, such as breast, ovary, uterus, cervix, lung, and testis, has been described. Sizeable gastrointestinal polyps may also give rise to intussusception/obstruction.

KEYWORDS: Peutz-Jeghers, Polyp, Hamartoma

INTRODUCTION: Peutz-Jeghers polyps are uncommon hamartomatous polyps that occur as a part of a rare autosomal dominant Peutz-Jeghers syndrome. The eponym Peutz Jeghers syndrome was originally put forward in 1954 by Bruwer et al. who based the name on the work of Peutz, who described a family with autosomal dominant inheritance of gastrointestinal polyposis and pigmented mucous membranes, and Jeghers who defined the coexistence of mucocutaneous pigmentation and gastrointestinal polyposis as a distinct clinical entity. ${ }^{3}$ The estimated incidence is 1 in 1,20,000 (50\% are familial and 50\% are new mutations). ${ }^{1}$ Male to female ratio is $1: 1 .^{1}$ Pigmented lesions are present in the first years of life and may fade at puberty, ${ }^{1}$ except for lesions on the buccal mucosa making the diagnosis possible in pediatric patients with a high level of suspicion.

CASE SUMMARY: 12 year old Indian female presented with chief complaints of abdominal pain, vomiting and constipation. No other family members had similar complaints. On examination, there was guarding, rigidity and tenderness of the abdomen with sluggish bowel sounds. Other findings included pigmentation of the lips and periorbital area. USG abdomen shows iso to hypoechoic lesion measuring $4 \times 3 \mathrm{~cm}$ in lower epigastric region, no evidence of intussusception or obstruction. On laporotomy, polyps were seen in the jejunum. Grossly, a segment of intestine was received showing two pedunculated polyps, largest measuring $4 \mathrm{~cm}$.Microscopically, multiple sections revealed tree like branching of muscularis mucosae with glandular proliferation and scattered inflammatory cell infiltrate in between the glands.

DISCUSSION: Peutz-Jeghers polyps are hamartomatous polyps that involve the mucosal epithelium, lamina propria and muscularis propria, associated with mutation of gene STK11(LKB1) on chromosome 19p13.3.4 Peutz Jeghers Syndrome is characterized by multiple 
hamartomatous polyps scattered throughout the gastrointestinal tract and melanotic mucosal and cutaneous pigmentation around the lips, oral mucosa, face, genitilia and palmar surface of hands. ${ }^{3}$ Mucocutaneous pigmented lesions are seen in around $95 \%$ of patients and may be the first clue to an individual having Peutz Jeghers Syndrome. ${ }^{3}$ Lesions tend to arise in infancy, occurring around the mouth, nostrils, perianal area, fingers and toes, and the dorsal and volar aspects of hands and feet. ${ }^{3}$ They may fade after puberty but tend to persist in the buccal mucosa. ${ }^{3}$ Diagnostic Criteria for Peutz-Jeghers Syndrome (PJS) include (1) Three or more histologically confirmed Peutz-Jeghers polyps, or (2) Any number of Peutz-Jeghers polyps with a family history of PJS, or (3) Characteristic prominent mucocutaneous pigmentation with a family history of PJS, or (4) Any number of Peutz-Jeghers polyps and characteristic prominent mucocutaneous pigmentation. ${ }^{1}$ Microscopically, a Peutz-Jeghers polyp arising in the small intestine is typically characterized by smooth muscle bundles of varying thickness extending in an arborizing arrangement up into the "head" of the polyp.1,3 Mortality may be due to two resons. Firstly, large polyps may cause abdominal pain and intussusception, sometimes causing bowel obstruction and severe gastrointestinal bleeding leading to anaemia. ${ }^{4}$ Secondly, in addition to an elevated risk of gastrointestinal cancers, an increased risk of cancers at other sites, such as breast, ovary, uterus, cervix, lung, and testis, has been described. ${ }^{5}$ Rare tumors have also been attributed to Peutz-Jeghers syndrome, including testicular sex cord and Sertoli cell tumors, leading to sexual precocity and gynecomastia, ovarian sex cord tumors with annular tubules, and adenoma malignum of the cervix. ${ }^{5}$ The cumulative lifetime risk for cancer in patients with PJS is 93\%; 18 times greater than expected in the general population. ${ }^{2}$ PeutzJeghers syndrome has been linked to a mutation of the STK11 (LKB1) gene on chromosome 19, which is present in $20 \%$ to $70 \%$ of affected individuals with a family history and $14 \%$ to $50 \%$ of affected individuals with no family history of Peutz-Jeghers syndrome. ${ }^{2}$

CONCLUSION: Peutz-Jeghers polyp is rare non-neoplastic hamartomatous polyp of intestine. Peutz Jeghers syndrome is an autosomal dominant condition defined by the development of characteristic polyps throughout the gastrointestinal tract and mucocutaneous pigmentation. Diagnosis is important as this condition is associated with malignancy of ovary, uterus, pancreas, lung and breast. Also patients are at an increased risk of intussusceptions which is a common cause of mortality.

As Peutz-Jeghers Syndrome is rare in India, a case report has been sent for publication.

\section{REFERENCES:}

1. Fenoglio-Preiser CM, Noffsinger AE, Stemmermann GN, Lantz PE, Isaacson PG. Gastointestinal Pathology: An Atlas and Text. 3rd Ed: Lippincott Williams \& Wilkins; 2008. Chapter 12, Polyposis and Hereditary Cancer Syndromes;692-734.

2. Burkart AL, Sheridan T, Lewin M, Fenton H, Ali NJ, Elizabeth M. Do sporadic PeutzJeghers polyps exist? Experience of a large teaching hospital. Am J Surg Pathol. 2007 Aug;31(8):1209-1214.

3. Beggs AD, Latchford AR, Vasen HFA, Moslein G,Alonso A, Aretz S et al. Peutz-Jeghers syndrome: a systematic review and recommendations for management. Gut. 2010;59:975-986.

4. Amos CI, Keitheri-Cheteri MB, Sabripour M, Wei C, McGarrity TJ, Seldin MF, Nations L, Lynch PM, Fidder HH, Friedman E, Frazier ML. Genotype-phenotype correlations in Peutz-Jeghers syndrome. J Med Genet 2004;41:327-333. 
5. Hearle N, Schumacher V, Menko FH, Olschwang S, Boardman LA, Gille JJP. Frequency and Spectrum of Cancers in the Peutz-Jeghers Syndrome. Clin Cancer Res. 2006 May 15;12(10):3209-3215.

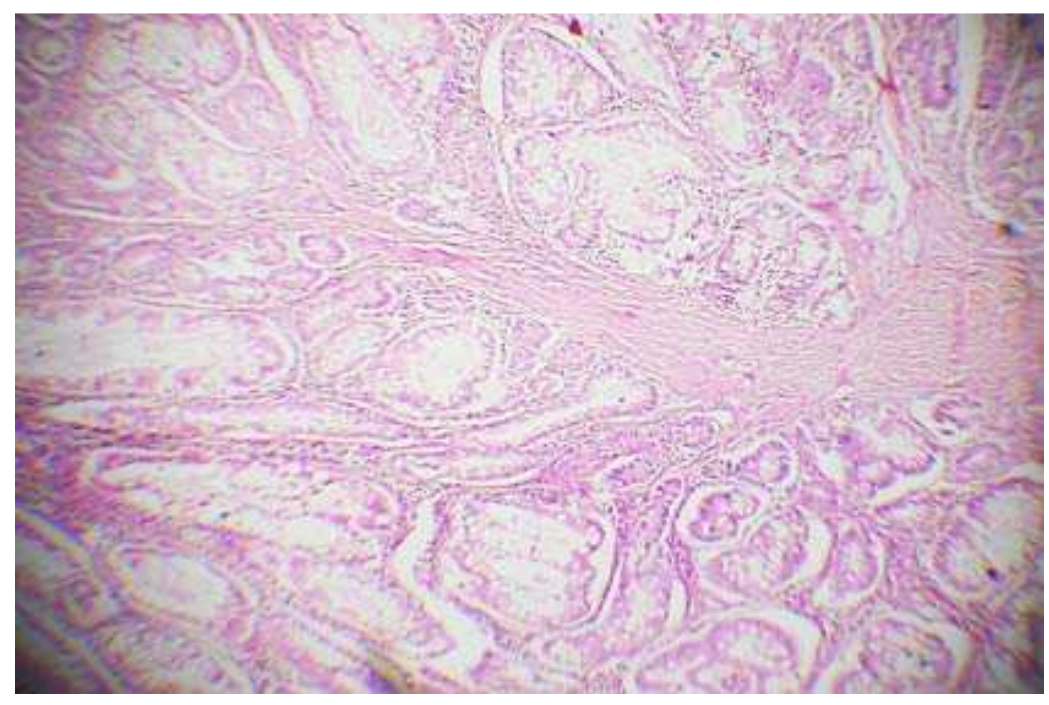

\section{Peutz Jeghers Polyp 10x}

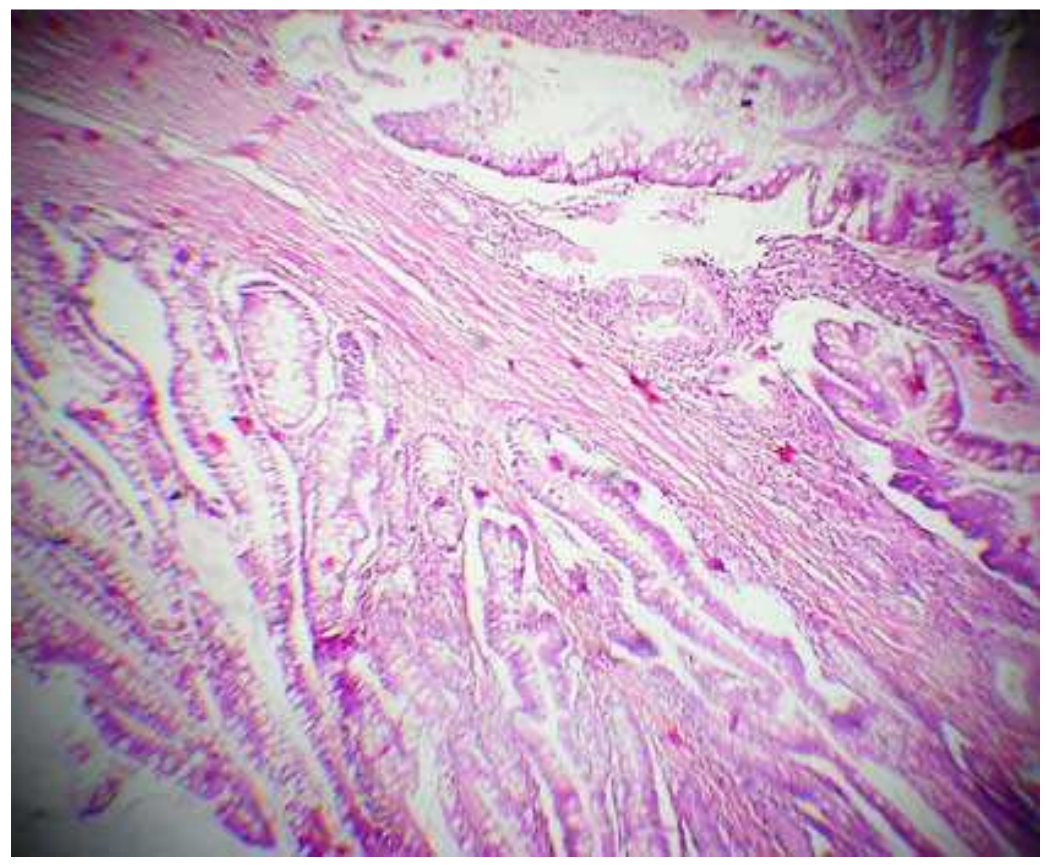

peutz jeghers polyp 10x.. 


\section{CASE REPORT}
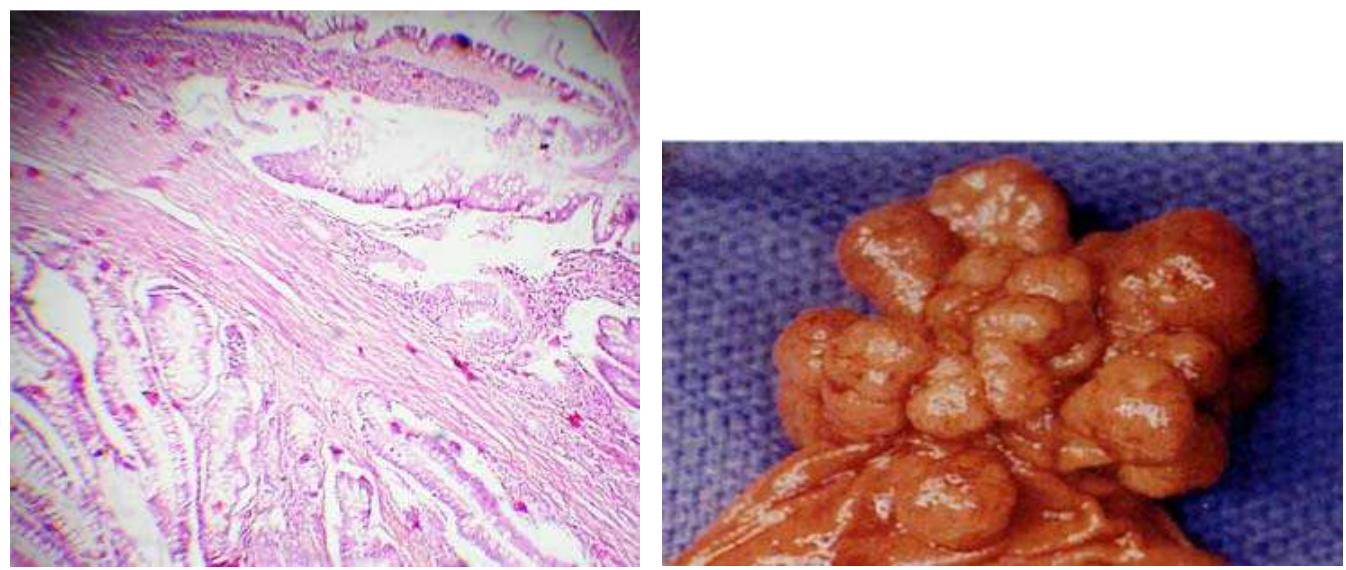

Peutz Jeghers Polyp 40x

Peutz Jeghers Polyp - Gross

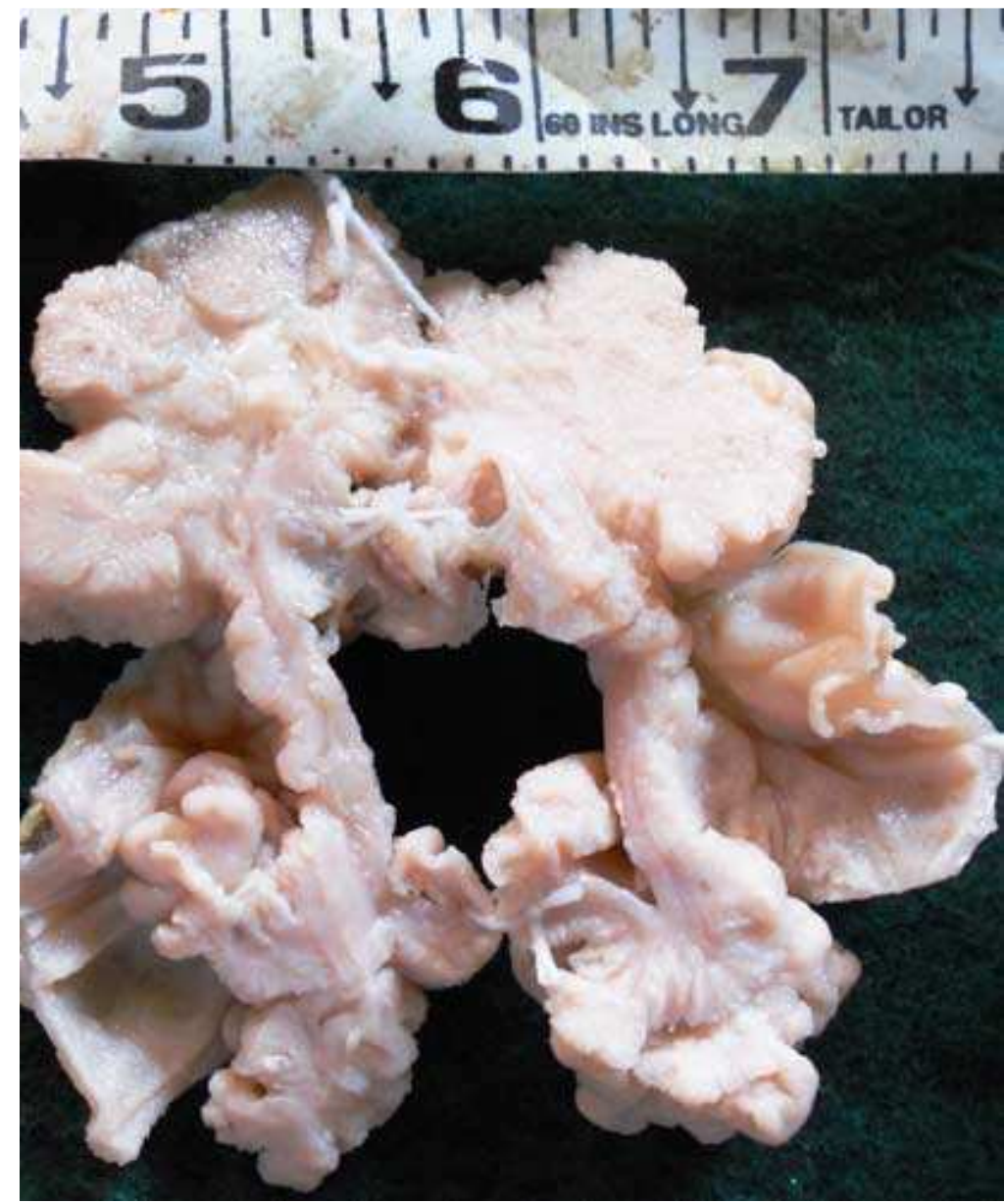

Specimen showing gross cut surface of Peutz Jeghers Polyp 